\title{
Efficacy, safety, and patient acceptability of elvitegravir/cobicistat/emtricitabine/tenofovir in the treatment of HIVIAIDS
}

This article was published in the following Dove Press journal:

Patient Preference and Adherence

24 August 2015

Number of times this article has been viewed

\author{
Roberta Prinapori' \\ Antonio Di Biagio² \\ 'Infectious Diseases, University \\ of Genoa, Genoa, Italy; ${ }^{2}$ Unit of \\ Infectious Diseases, IRCCS AOU San \\ Martino-IST, Genoa, Italy
}

\begin{abstract}
The fixed-dose combination (FDC) elvitegravir/cobicistat/emtricitabine/tenofovir (EVG/c/FTC/TDF) is a once-daily, single-tablet regimen containing an integrase strand transfer inhibitor and a pharmacoenhancer (cobicistat) associated with two nucleos(t)ide reverse transcriptase inhibitors. It is approved as the preferred regimen and as the first-line combined antiretroviral therapy in treatment-naïve patients with HIV infection. Two large trials, 102Study and 103-Study, demonstrated that EVG/c/FTC/TDF was not inferior to efavirenz/FTC/ TDF and ritonavir-boosted atazanavir in association with FTC/TDF, in terms of virological suppression and immunological reconstitution through week 144. Also, simplification arms containing EVG/c/FTC/TDF reached noninferiority in comparison with a nonnucleoside reverse transcriptase inhibitor, or a protease inhibitor, or a raltegravir-based regimen. Furthermore, EVG/c/FTC/TDF exhibited an excellent tolerability profile, with a safer lipid profile, and despite the indication of its use in subjects with an estimated creatinine clearance $>70 \mathrm{~mL} / \mathrm{min}$, recent data demonstrated that EVG/c/FTC/TDF determined a reduction in estimated glomerular filtration rate (GFR) but not a reduction of actual GFR. Moreover, in a cohort of naïve patients with pretreatment mild-to-moderate renal impairment, GFR decrease was noted as early at week 2, after which it generally stabilized and was nonprogressive through week 48. The FDC's efficacy and good tolerability enable EVG/c/FTC/TDF to meet the patients' needs, improving adherence and quality of life, which are among the most important factors affecting the therapeutic efficacy of an antiretroviral regimen. This paper describes the evidence making EVG/c/FTC/ TDF a new therapeutic opportunity for different HIV-infected patients.
\end{abstract}

Keywords: HIV, once daily, elvitegravir, single-tablet regimen, fixed-dose combination, adherence

\section{Introduction}

Thanks to the introduction of new drugs or new fixed-dose combinations (FDCs) in the recent years, the combined antiretroviral treatment (cART) is now able to ensure almost excellent efficacy and tolerability profiles in HIV-infected patients.

Simplification of dosing frequency and reduction of pill burden are the key features for new regimens, aimed at improving adherence and quality of life of people living with HIV. As recently reported by AIDS Clinical Trials Group Study A5257, the integrase strand transfer inhibitor (INSTI)-containing regimens are of equivalent efficacy and have high rate of virologic suppression than that of protease inhibitor regimen (PI) (atazanavir/ritonavir and darunavir [DRV]/ritonavir), but have more favorable tolerability profile and few adverse effects (lipids and bilirubin). ${ }^{1}$

INSTI drugs prevent or inhibit the binding of the preintegration complex to host cell DNA, thus terminating the integration step of HIV replication ${ }^{2}$ and resulting in a rapid
Correspondence: Antonio Di Biagio Unit of Infectious Diseases, IRCCS AOU San Martino-IST, Largo R. Benzi I0, I6I32 Genoa, Italy

Tel +39010 5554651

Fax +39010 5556794

Email antonio.dibiagio@hsanmartino.it 
early-phase decay of plasma HIV-RNA. ${ }^{3}$ As HIV integrase has no counterpart in host cells, INSTIs do not interfere with common cellular process, thus ensuring a safer profile. ${ }^{4}$

For these reasons, all three currently available INSTIs are included now among the recommended regimens and, in general, should be selected for most patients. ${ }^{1,5}$ Furthermore, when choosing between regimens of similar efficacy and tolerability, it is suggested to use once-daily regimens for both treatment-naïve patients beginning cART and experienced patients receiving complex or poorly tolerated regimens and to use FDCs and single-tablet regimens (STRs) to decrease pill burden. ${ }^{6}$

Among STR formulations, elvitegravir/cobicistat/ emtricitabine/tenofovir disoproxil fumarate (EVG/c/FTC/ $\mathrm{TDF}$ ) is a relatively new combination. It was approved by Food and Drug Administration in August 2012 in a once-a-day FDC to treat cART-naïve patients or to switch cART-experienced patients. Composed of an INSTI (EVG), a pharmacoenhancer (cobicistat), and two reverse transcriptase inhibitors (TDF and FTC), it is the first INSTI-based STR available.

The aim of this review is to evaluate the clinical usefulness, defined as the quality of having medical utility and especially practical worth or applicability in clinical practice, of the combination of EVG/c/FTC/TDF in the management of HIV infection.

\section{Current options for naïve and experienced patients}

The 2015 US Department of Health and Human Services

(DHHS) guidelines recommend a combination of two reverse transcriptase inhibitors (NRTI) plus a ritonavir-boosted DRV, or an INSTI for the initial treatment of HIV-1-infected adults and adolescents (Table 1). ${ }^{5}$

INSTIs are now the standard of care for naïve HIVinfected individuals due to their efficacy and safety profile. Three different integrase inhibitors were approved: dolutegravir (DTG), EVG/c, and raltegravir (RAL). Tenofovir/ emtricitabine and abacavir/lamivudine $(\mathrm{ABC} / 3 \mathrm{TC})$ were the NRTIs preferred in first-line treatment.

Despite the high efficacy of the currently preferred cART regimens ( $75 \%$ over 99 weeks), than the alternative regimens (65\%, difference 10\%; 95\% confidence interval 7.6-15.4; $P<0.001)$, ${ }^{7}$ cART does not eradicate the HIV infection, and must be continued indefinitely.

The persistence of first-line cART regimen is essential for the management of HIV infection. ${ }^{8}$

In Western countries, adverse events and treatment failure were the two most common reasons for first-line cART discontinuation or modification. ${ }^{9}$ In addition, a greater number of medications within a regimen and more frequent dosing were associated with early discontinuations. ${ }^{10}$ These data are supported by several studies in which simple, oncedaily cART regimens demonstrate high levels of adherence and treatment satisfaction, resulting in persistent viral suppression. ${ }^{11-13}$

Currently, four FDCs are approved for the treatment of HIV-1 infection: Atripla ${ }^{\circledR}$ (efavirenz FTC/TDF; BristolMyers Squibb Company, Princeton, NJ, USA), Complera ${ }^{\circledR} /$ Eviplera $^{\circledR}$ (FTC/TDF/rilpivirine; Gilead Sciences, Inc.

Table I DHHS recommendations on preferred and alternative regimen for first-line antiretroviral therapy ${ }^{5}$

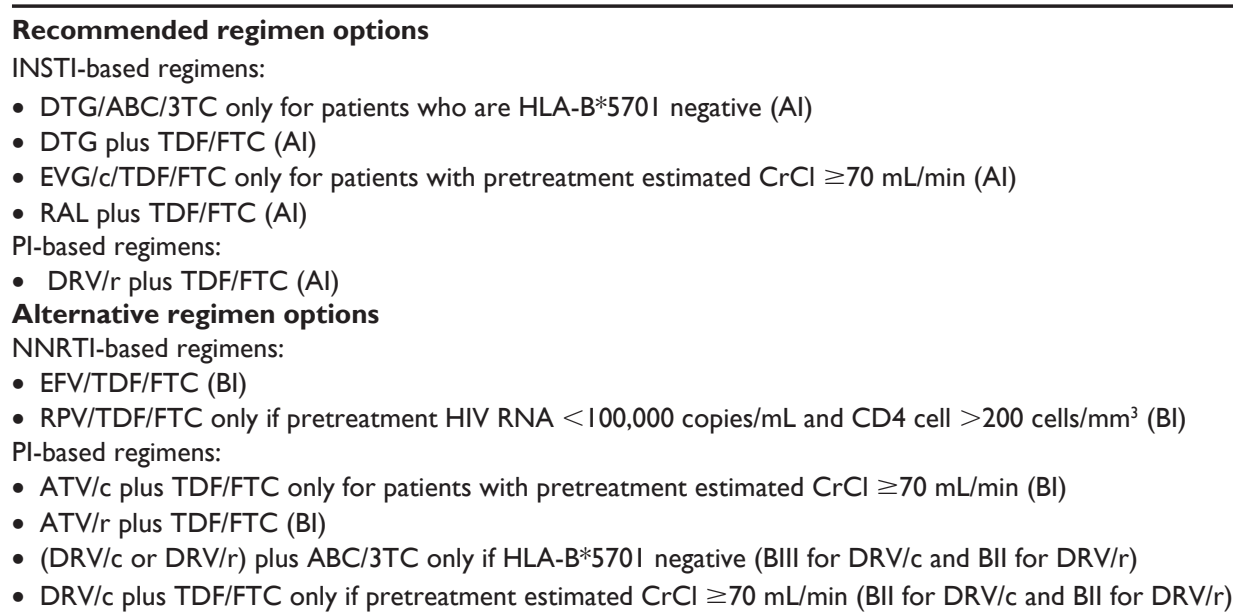

Notes: Evidence rating is either 'A' for strong recommendation, or 'B' for moderate recommendation. Quality of evidence classified as 'l' being one or more randomized trials with clinical outcomes and/or validated, 'll' being one or more well-designed, non-randomized trials or observational cohort studies with long-term clinical outcomes, and 'III' being expert opinion. *Indicate allelic variants.

Abbreviations: DHHS, US Department of Health and Human Services; INSTI, integrase strand transfer inhibitor; DTG, dolutegravir; ABC/3TC, abacavir/lamivudine; TDF/FTC, tenofovir/emtricitabine; EVG/c, elvitegravir/cobicistat; RAL, raltegravir; CICr, creatinine clearance; PI, protease inhibitors; DRV/r, darunavir/ritonavir; EFV, efavirenz; RPV, rilpilvirine; ATV/c, atazanavir/cobicistat; ATV/r, atazanavir/ritonavir; DRV/c, darunavir/cobicistat. 
Foster City, CA, USA), Stribild ${ }^{\circledR}$ (EVG/c/FTC/TDF; Gilead Sciences, Inc), and only in the United States and Canada, Triumeq ${ }^{\circledR}$ (ABC/3TC/DTG; Pfizer, Inc, New York, NY, USA).

The two INSTI-containing STRs are the preferred regimen, both with a footnote, EVG/c/FTC/TDF only for patients with pretreatment estimated creatinine clearance $(\mathrm{CrCL}>70$ $\mathrm{mL} / \mathrm{min}$ ) and $\mathrm{ABC} / 3 \mathrm{TC} / \mathrm{DTG}$ only for patients who are HLA-B*5701 negative.

\section{Efficacy profile in naïve patients}

The efficacy of EVG/c/FTC/TDF in cART-naïve patients with HIV infection was evaluated in two large randomized, Phase III, double-blind active-controlled trials.

In the 102-Study, patients received once-daily EVG/c/ FTC/TDF or efavirenz/FTC/TDF. In the 103-Study, patients received once-daily EVG/c/FTC/TDF or ritonavir-boosted atazanavir in association with FTC/TDF. In both studies, the primary end point was HIV-1 RNA $<50$ copies/mL at week $48,{ }^{14,15}$ with secondary analysis at week $96^{16,17}$ and week $144 .^{18,19}$

EVG/c/FTC/TDF was noninferior to comparators regimens in terms of virological suppression and immunological reconstitution through week 144 . The mean increase of lymphocyte $\mathrm{T}-\mathrm{CD} 4^{+}$cell count at most time points were similar in all groups. However, at week 48, mean increase in lymphocyte $\mathrm{T}-\mathrm{CD} 4^{+}$cell count was significantly higher in the EVG/c/FTC/TDF group than in the efavirenz/FTC/TDF $\operatorname{arm}(239$ vs 206 cells $/ \mu \mathrm{L} ; P=0.009) .{ }^{14}$

The rate of resistance development to EVG/c/FTC/TDF was low in the $102-$ Study and $103-$ Study ( $<2 \%$ treated). The most common pattern of resistance was $\mathrm{M} 184 \mathrm{~V}$ in reverse transciptase and E92Q in INSTI. There was no selection pressure from cobicistat for protease substitutions. ${ }^{18,19}$

\section{Efficacy profile in simplification}

The efficacy of EVG/c/FTC/TDF in simplification was evaluated in three different trials. STRATEGY-NNRTI ${ }^{20}$ and STRATEGY-PI ${ }^{21}$ are 96-week, international, multicenter, randomized, open-label, Phase IIIb, noninferiority trials enrolling adults ( $\geq 18$ years) with HIV-1 and plasma HIV-RNA $<50$ copies/mL for at least 6 months on a(n) nonnucleoside reverse transcriptase inhibitor (NNRTI)based regimen or a ritonavir-boosted PI (atazanavir, DRV, lopinavir, fosamprenavir, or saquinavir) plus FTC/TDF. Patients were randomly assigned (2:1) to switch to EVG/c/ FTC/TDF. Key eligibility criteria included no history of virological failure and an estimated glomerular filtration rate
(GFR) of $70 \mathrm{~mL} / \mathrm{min}$ or greater. The primary end point was the proportion of participants with plasma viral loads $<50$ copies/mL at week 48 . At 48 weeks, 93\% subjects compared to $88 \%$ in STRATEGY-NNRTI $(P=0.066)$, and $93.8 \%$ subjects compared to $87.1 \%$ in STRATEGY-PI $(P=0.025)$ maintained viral suppression. An innovative approach examined the switching of 48 subjects from twice-daily RAL plus FTC TDF to once-daily EVG/c/FTC/TDF. ${ }^{22}$ All of the enrolled subjects maintained HIV-RNA $<50$ copies/mL throughout the 48 weeks of treatment.

These data taken together support the fact that EVG/c/ TDF/FTC can be a viable candidate for those patients seeking to discontinue their current drug regimen.

\section{Safety profile}

$\mathrm{EVG} / \mathrm{c} / \mathrm{FTC} / \mathrm{TDF}$ is well tolerated; the most common adverse events reported in registration trials were diarrhea, nausea, upper respiratory infection, and headache (Table 2). ${ }^{14-19}$ The use of EVG/c/TDF/FTC is limited by several drug interactions because of the fact that EVG is metabolized primarily by CYP3A enzymes; as a result, CYP3A inducers or inhibitors may alter EVG plasma concentrations. Moreover, because of inhibition of CYP3A by cobicistat, EVG/c/FTC/TDF interacts with a number of medications that are metabolized by this enzyme. ${ }^{23}$

Furthermore, cobicistat, like cimetidine, trimethoprimsulfamethoxazole, telaprevir, ritonavir, and DTG, increases serum creatinine by blocking its tubular secretion. This results in a reduction in estimated GFR but not a reduction of actual GFR, as demonstrated in prior iohexol studies with cobicistat. This inhibition is mediated via blockage of the cationic transporters, including the efflux pump multidrug and toxin extrusion protein 1 in the renal proximal tubule, and is reversible. ${ }^{24}$

In the 102-Study and 103-Study, an increase in creatinine with EVG/c/FTC/TDF was observed, and it occurred mostly by week 4 . Thereafter, creatinine levels stabilized and were nonprogressive through week 144 . These changes occurred in both groups ( $<$ and $\geq 50$ years). ${ }^{18,19}$ In an in vitro study conducted in various renal cell and tissue models aimed at investigating the potential for a renal drug-drug interaction between TDF and cobicistat, Stray et $\mathrm{al}^{25}$ observed no increase in the accumulation of tenofovir (TFV), the hydrolyzed form of the prodrug TDF, in freshly isolated human renal cortex tissue or renal proximal tubule cells, in the presence of cobicistat and demonstrated that cobicistat and TFV interacted primarily with distinct renal transporters indicating a low potential for pharmacokinetic renal drug-drug interaction. 
Table 2 Most common adverse events reported in registration trials

\begin{tabular}{|c|c|c|c|c|}
\hline & \multicolumn{2}{|c|}{ I 02-Study $(n=348)$} & \multicolumn{2}{|c|}{ 103-Study (n=353) } \\
\hline & Week 96 & Week I44 & Week 96 & Week I44 \\
\hline \multicolumn{5}{|c|}{ AEs (all grades) $(\%)$ reported in $\geq 10 \%$ of patients } \\
\hline Diarrhea & 25 & 26 & 25 & 22 \\
\hline Nausea & 22 & 23 & 21 & 22 \\
\hline Upper respiratory tract infection & 21 & 26 & 20 & 24 \\
\hline Headache & 16 & 18 & 17 & 18 \\
\hline Nasopharyngitis & 10 & II & 10 & 14 \\
\hline Depression & 12 & 15 & 10 & 12 \\
\hline Fatigue & 13 & 15 & 15 & 17 \\
\hline Bronchitis & 8 & 11 & 10 & 13 \\
\hline Sinusitis & 9 & 11 & 7 & 8 \\
\hline Cough & 7 & 10 & 8 & 10 \\
\hline Rash & 7 & 9 & 9 & 8 \\
\hline \multicolumn{5}{|c|}{ AEs (\%) leading to study drug discontinuation in $>$ I patient } \\
\hline Renal events & 2.0 & 2.2 & 0.8 & 1.4 \\
\hline Hepatitis $C$ virus & & & 0.3 & 0.6 \\
\hline Diarrhea & & & 0.6 & 0.6 \\
\hline Pyrexia & & & 0.6 & 0.6 \\
\hline Fatigue & 0.3 & 0.3 & 0.3 & 0.3 \\
\hline Depression & 0.3 & 0.3 & & \\
\hline Nausea & & & 0.3 & 0.3 \\
\hline Vomiting & & & 0.3 & 0.3 \\
\hline
\end{tabular}

Abbreviation: AEs, adverse events.

The safety renal profile of EVG/c/FTC/TDF has also been recently investigated by Post et $\mathrm{al}^{26}$ in a 96-week, phase III, open-label, multicenter study in which they demonstrated a renal safety profile of EVG/c/FTC/TDF in 33 treatment-naïve patients with pretreatment mild-to-moderate renal impairment: decreases in median value of $\mathrm{CrCL}$ and GFR were noted as early at week 2 , after which they generally stabilized and were nonprogressive through week 48. Furthermore, in a Phase I study of EVG and cobicistat in patients with severe renal impairment $(\mathrm{CrCL}<30 \mathrm{~mL} / \mathrm{min})$, no clinically relevant differences in EVG or cobicistat exposures were seen. ${ }^{27}$

As for the lipid profile in 102-Study, EVG/c/FTC/TDF showed a smaller median increases in total cholesterol $(P=0.007)$, fasting low-density lipoprotein cholesterol (LDL-c) $(P=0.007)$, and fasting high-density lipoprotein cholesterol (HDL-c) $(P=0.021)$ at week 144 than EFV/ FTC/TDF; increases in triglycerides and changes in total cholesterol to HDL ratio were similar in the two groups. ${ }^{18}$ In the 103-Study at week $144,{ }^{19}$ the median change from baseline fasting triglycerides was numerically higher but not significant $(P=0.24)$. There were no significant treatment differences in change from baseline through week 144 in median LDL-c, HDL-c, or fasting total cholesterol to fasting HDL-c cholesterol ratio. In the 103-Study, a bone mineral quality assessment was also performed. At week 144, the mean percent decrease from baseline in spine bone mineral density (BMD) and in hip BMD were $-1.43 \%$ and $-2.83 \%$ in the EVG group and $-3.68 \%$ and $-3.77 \%$ in the atazanavir group ( $P=0.018$ and 0.23 , respectively).

Another recently highlighted advantage of the INSTI class of drugs is its ability in reducing levels of inflammatory and coagulation biomarkers and reducing the level of immune activation both in naïve and experienced patients. ${ }^{28,29}$ In the Spiral study, a switch from PI-based therapy to a RALcontaining regimen in patients with suppressed viremia led to a decrease in biomarkers associated with inflammation, insulin resistance, and hypercoagulability. ${ }^{30}$ Furthermore, it has been recently noted that switching to RAL-based regimens may be associated with a decrease of HIV reservoir, as measured by total peripheral blood mononuclear cells' HIV DNA. ${ }^{31}$ As observed for RAL-based cART, EVG/c/FTC/ TDF had led to greater decreases in markers of monocyte activation and vascular inflammation, in particular, sCD14, hsCRP, and Lp-PLA2 levels, than EFV/FTC/TDF in cARTnaïve HIV-infected adults. ${ }^{32}$

\section{Patient acceptability}

When effective cART was first developed almost two decades ago, adherence was particularly challenging because patients had to consume handfuls of pills, often with substantial toxicity, multiple times per day. ${ }^{33}$ The introduction of STR strategies has been associated with an improvement in 
Table 3 Questions included in the treatment ease questionnaire

Treatment ease questions (HIV treatment satisfaction questionnaire)

Score per question -3 (much less satisfied) to 3 (much more satisfied)

Treatment ease score range -15 to 15

I. How convenient have you been finding your treatment to be recently?

2. How satisfied are you with the demands made by your current treatment?

3. How satisfied are you with the extent to which the treatment fits in with your lifestyle?

4. How flexible have you been finding your treatment to be recently?

5. How satisfied are you with your understanding of your HIV?

adherence rate. ${ }^{34}$ As emerged in the ADONE (ADherence to ONE pill) study ${ }^{35}$ which aimed at verifying the effect of a reduced number of pills on adherence and quality of life in HIV-infected patients on cART, a one-pill once-a-day cART increased significantly the adherence rate at 1 month after switching to STR $(P<0.01)$ that was maintained throughout the study. Quality of life, which significantly influenced adherence $(P<0.0001)$, improved over time $(P=0.042)$. Furthermore, patient's opinion in terms of patient's preferences concerning tolerability, convenience, simplicity, and efficacy was significantly in favor of the FDC.

In two subgroup analysis of STRATEGY studies, ${ }^{35,36}$ the patient's satisfaction was investigated using an Ease Score. Questions included in the questionnaire are listed in Table 3. In the NNRTI-STRATEGY subgroup analysis, 59 patients switched to EVG/c/FTC/TDF, and 37 continued a non-EFV NNRTI (27 nevirapine, ten rilpilvirine) with FTC/TDF. Switch to EVG/c/FTC/TDF was associated with a higher treatment ease (convenience, flexibility, demand, lifestyle, understanding) score (range: -15 to 15 ) at week 4 (median: 14 vs $11 ; P=0.047$ ) and week 24 (median: 14 vs $12.5 ; P=0.038$ ) than patients who continued their nevirapine- or rilpilvirinebased cART. In the PI STRATEGY subgroup analysis, 113 subjects switched to EVG/c/FTC/TDF; 60 continued a ritonavir-boosted DRV with FTC/TDF. An increased satisfaction with the ease of therapy for subjects who simplified their multitablet DRV-based regimen to the STR EVG/c/FTC/ TDF was observed at week 4 (median: 12 vs $9 ; P=0.006$ ) and week 24 (median: 13 vs $8 ; P<0.001$ ).

\section{Resource-limited setting}

The use of EVG/c/FTC/TDF offers the potential to improve the simplicity, safety, and efficacy of first-line antiretroviral therapy in resource-limited settings. However, Stribild $^{\circledR}$ (Gilead Sciences, Inc) registration in the developing world is still not comprehensive. In March 2015, its approval was confirmed by only nine developing countries. ${ }^{37}$ No data are reported on interactions with drugs to treat common coinfections including tuberculosis and malaria.

\section{Present scenario and future perspectives}

$\mathrm{EVG} / \mathrm{c} / \mathrm{FTC} / \mathrm{TDF}$ has been shown to be effective and safe in adults patients with $\mathrm{CrCl}>70 \mathrm{~mL} / \mathrm{min}$. The single tablet and FDC offer advantages over more complex drug regimens, and improve adherence and persistence.

The privileged position in the different international guidelines, and the clinical use in the next few years, will enable EVG/c/FTC/TDF to have a strategic role in the management of patients with HIV infection.

\section{Disclosure}

The authors report no conflicts of interests in this work.

\section{References}

1. Lennox JL, Landovitz RJ, Ribaudo HJ, et al. Efficacy and tolerability of 3 nonnucleoside reverse transcriptase inhibitor-sparing antiretroviral regimens for treatment-naive volunteers infected with HIV-1: a randomized, controlled equivalence trial. Ann Intern Med. 2014;161(7):461-471.

2. Hazuda DJ, Felock P, Witmer M, et al. Inhibitors of strand transfer that prevent integration and inhibit HIV-1 replication in cells. Science. 2000;287(5453):646-650.

3. Stephan C, Baldauf HM, Barry J, et al. Impact of raltegravir on HIV-1 RNA and DNA forms following initiation of antiretroviral therapy in treatment-naive patients. J Antimicrob Chemother. 2014;69(10) 2809-2818.

4. Pommier Y, Johnson AA, Marchand C. Integrase inhibitors to treat HIV/AIDS. Nat Rev Drug Discov. 2005;4(3):236-248.

5. Guidelines for the Use of Antiretroviral Agents in HIV-1-Infected Adults and Adolescents (Downloaded from http://aidsinfo.nih.gov/guidelines on 4/15/2015). Available from: http://aidsinfo.nih.gov/contentfiles/ lvguidelines/adultandadolescentgl.pdf. Accessed July 31, 2015.

6. Thompson MA, Mugavero MJ, Amico KR, et al. Guidelines for improving entry into and retention in care and antiretroviral adherence for persons with HIV: evidence-based recommendations from an International Association of Physicians in AIDS Care panel. Ann Intern Med. 2012;156(11):817-833.

7. Lee FJ, Amin J, Carr A. Efficacy of initial antiretroviral therapy for HIV-1 infection in adults: a systematic review and meta-analysis of 114 studies with up to 144 weeks' follow-up. PLoS One. 2014;9(5):e97482.

8. Di Biagio A, Prinapori R, Giannerelli D, et al. Duration of first-line antiretroviral therapy with tenofovir and emtricitabine combined with atazanavir/ritonavir, efavirenz or lopinavir/ritonavir in the Italian ARCA cohort. J Antimicrob Chemother. 2013;68(1):200-205.

9. O'Brien ME, Clarks RA, Besch CL, et al. Patterns and correlates of discontinuation of the initial HAART regimen in an urban outpatient cohort. J Acquir Immune Defic Syndr. 2003;34(4):407-414.

10. Willig JH, Abroms S, Westfall AO, et al. Increased regimen durability in the era of once-daily fixed-dose combination antiretroviral therapy. AIDS. 2008;22(15):1951-1960.

11. Jayaweera D, Dejesus E, Nguyen KL, et al. Virologic suppression, treatment adherence, and improved quality of life on a once-daily efavirenz-based regimens in treatment naïve HIV-1 infected patients over 96 weeks. HIV Clin Trials. 2009;10: 375-384. 
12. Maggiolo F, Migliorino M, Maserati R, et al. Once-a day treatment for HIV infection. Final 48-week results. Presented at: 8th Conference on Retroviruses and Opportunistic Infections, February 4-8, 2001, Chicago, IL [Abstract 320].

13. Nachega JB, Parienti JJ, Uthman OA, et al. Lower pill burden and oncedaily antiretroviral treatment regimens for HIV infection: a meta-analysis of randomized controlled trials. Clin Infect Dis. 2014;58:1297-1307.

14. Sax PE, DeJesus E, Mills A, et al. GS-US-236-0102 study team. Coformulated elvitegravir, cobicistat, emtricitabine, and tenofovir versus co-formulated efavirenz, emtricitabine, and tenofovir for initial treatment ofHIV-1 infection: a randomised, double-blind, phase 3 trial, analysis of results after 48 weeks. Lancet. 2012;379(9835):2439-2448.

15. DeJesus E, Rockstroh JK, Henry K, et al; GS-236-0103 Study Team. Co-formulated elvitegravir, cobicistat, emtricitabine, and tenofovir disoproxil fumarate versus ritonavir-boosted atazanavir plus co-formulated emtricitabine and tenofovir disoproxil fumarate for initial treatment of HIV-1 infection: a randomised, double-blind, phase 3, non-inferiority trial. Lancet. 2012;379(9835):2429-2438.

16. Zolopa A, Sax PE, DeJesus E, et al; GS-US-236-0102 Study Team. A randomized double-blind comparison of coformulated elvitegravir/cobicistat/ emtricitabine/tenofovir disoproxil fumarate versus efavirenz/emtricitabine/ tenofovir disoproxil fumarate for initial treatment of HIV-1 infection: analysis of week 96 results. J Acquir Immune Defic Syndr. 2013;63(1):96-100.

17. Rockstroh JK, DeJesus E, Henry K, et al; GS-236-0103 Study Team. A randomized, double-blind comparison of coformulated elvitegravir/ cobicistat/emtricitabine/tenofovir DF vs ritonavir-boosted atazanavir plus coformulated emtricitabine and tenofovir DF for initial treatment of HIV-1 infection: analysis of week 96 results. J Acquir Immune Defic Syndr. 2013;62(5):483-486.

18. Wohl DA, Cohen C, Gallant JE, et al. A randomized, double-blind comparison of single-tablet regimen elvitegravir/cobicistat/emtricitabine/ tenofovir DF versus single-tablet regimen efavirenz/emtricitabine/ tenofovir DF for initial treatment of HIV-1 infection: analysis of week 144 results. J Acquir Immune Defic Syndr. 2014;65(3):e118-e120.

19. Clumeck N, Molina JM, Henry K, et al; GS-236-0103 Study 96. A randomized, double-blind comparison of single-tablet regimen elvitegravir/ cobicistat/emtricitabine/tenofovir DF vs ritonavir-boosted atazanavir plus emtricitabine/tenofovir DF for initial treatment of HIV-1 infection: analysis of week 144 results. J Acquir Immune Defic Syndr. 2014;65(3):e121-e124.

20. Pozniak A, Markowitz M, Mills A, et al. Switching to coformulated elvitegravir, cobicistat, emtricitabine, and tenofovir versus continuation of non-nucleoside reverse transcriptase inhibitor with emtricitabine and tenofovir in virologically suppressed adults with HIV (STRATEGYNNRTI): 48 week results of a randomised, open-label, phase $3 \mathrm{~b}$ noninferiority trial. Lancet Infect Dis. 2014;14:590-599.

21. Arribas JR, Pialoux G, Gathe J, et al. Simplification to coformulated elvitegravir, cobicistat, emtricitabine, and tenofovir versus continuation of ritonavir-boosted protease inhibitor with emtricitabine and tenofovir in adults with virologically suppressed HIV (STRATEGY-PI): 48 week results of a randomised, open-label, phase $3 \mathrm{~b}$, non-inferiority trial. Lancet Infect Dis. 2014;14:581-589.

22. Mills A, Crofoot G, Ortiz R, et al. Switching from twice-daily raltegravir plus tenofovir disoproxil fumarate/emtricitabine to once-daily elvitegravir/cobicistat/emtricitabine/tenofovir disoproxil fumarate in virologically suppressed, HIV-1- infected subjects: 48 weeks data. HIV Clin Trials. 2014;15:51-56.

Patient Preference and Adherence

\section{Publish your work in this journal}

Patient Preference and Adherence is an international, peer-reviewed, open access journal that focuses on the growing importance of patient preference and adherence throughout the therapeutic continuum. Patient satisfaction, acceptability, quality of life, compliance, persistence and their role in developing new therapeutic modalities and compounds to optimize Submit your manuscript here: http://www.dovepress.com/patient-preference-and-adherence-journal
23. Mathias AA, West S, Hui J, Kearney BP. Dose-response of ritonavir on hepatic CYP3A activity and elvitegravir oral exposure. Clin Pharmacol Ther. 2009;85(1):64-70.

24. German P, Liu HC, Szwarcberg J, et al. Effect of cobicistat on glomerular filtration rate in subjects with normal and impaired renal function. J Acquir Immune Defic Syndr. 2012;61(1):32-40.

25. Stray KM, Bam RA, Birkus G, et al. Evaluation of the effect of cobicistat on the in vitro renal transport and cytotoxicity potential of tenofovir. Antimicrob Agents Chemother. 2013;57(10):4982-4989.

26. Post FA, Winston J, Andrade-Villanueva JF, et al; Study 118 Team. Elvitegravir/cobicistat/emtricitabine/tenofovir df in hiv-infected patients with mild-to-moderate renal impairment. $J$ Acquir Immune Defic Syndr. 2015;68(3):310-313.

27. German P. Parmacokinetics of elvitegravir and cobicistat in subjects with severe renal impairment. Poster presented at: 13th International Workshop on Clinical Pharmacology of HIV Therapy, April 16-18, 2012, Barcelona, Spain [Poster number 38].

28. Rockstroh JK, Lennox JL, Dejesus E, et al. Long-term treatment with raltegravir or efavirenz combined with tenofovir/emtricitabine for treatment-naive human immunodeficiency virus-1 infected patients: 156week results from STARTMRK. Clin Infect Dis. 2011;53:807-816.

29. Eron JJ, Young B, Cooper DA, et al. SWITCHMRK 1 and 2 investigators. Switch to a raltegravir-based regimen versus continuation of a lopinavirritonavir-based regimen in stable HIV-infected patients with suppressed viraemia (SWITCHMRK 1 and 2): two multicentre, double-blind, randomised controlled trials. Lancet. 2010;375(9712):396-407.

30. Martinez E, d'Albuquerque PM, Llibre J, et al; the SPIRAL Study Group. Changes of cardiovascular biomarkers in HIV-infected patients switching from ritonavir boosted protease inhibitor to raltegravir. AIDS. 2012;26:2315-2326.

31. Bianco C, Meini G, Rossetti B, et al. Switch to raltegravir-based regimens and HIV DNA decrease in patients with suppressed HIV RNA. J Int AIDS Soc. 2014;17(4 Suppl 3):19791.

32. Hileman CO, Kinley B, Scharen-Guivel V, et al. Differential reduction in monocyte activation and vascular inflammation with integrase inhibitor-based initial antiretroviral therapy. J Infect Dis. 2015;212(3): 345-354.

33. Gandhi M, Gandhi RT. Single-pill combination regimens for treatment of HIV-1 infection. N Engl J Med. 2014;371(3):248-259.

34. Parienti JJ, Bangsberg DR, Verdon R, Gardner EM. Better adherence with once-daily antiretroviral regimens: a meta-analysis. Clin Infect Dis. 2009;48(4):484-488.

35. Stellbrink HJ, Antinori A, Pozniak A, et al. Switch to stribild versus continuation of NVP or RPV with FTC and TDF in virologically suppressed HIV adults: a STRATEGY-NNRTI subgroup analysis. $J$ Int AIDS Soc. 2014;17(4 Suppl 3):19793

36. Arribas J, Rizzardini G, Arasteh K, et al. Simplification to Stribild vs continuation of RTV-boosted DRV with FTC and TDF in virologically suppressed HIV adults: a STRATEGY-PI subgroup analysis. J Int AIDS Soc. 2014;17(4 Suppl 3):19805.

37. Stribild ${ }^{\circledR}$ registration in the developing world. Available from: https://www.gilead.com/ /media/Files/pdfs/other/Stribild $\% 20$ Registration\%20102414.pdf. Accessed July 31, 2015. clinical outcomes for existing disease states are major areas of interest for the journal. This journal has been accepted for indexing on PubMed Central. The manuscript management system is completely online and includes a very quick and fair peer-review system, which is all easy to use. Visit http://www. dovepress.com/testimonials.php to read real quotes from published authors. 